\title{
Lost Packet Retransmission Mechanism Based on Hardware Acceleration in Wireless Sensor Networks
}

\author{
Yuanliang $\mathrm{Nie}^{1}$, Ping Song ${ }^{1, *}$, Cheng Yang ${ }^{2}$, Chuangbo Hao ${ }^{1}$ and Youtian Qie ${ }^{1}$ \\ ${ }^{1}$ Key Laboratory of Biomimetic Robots and Systems, Beijing Institute of Technology, Beijing, China \\ ${ }^{2}$ China Institute of Marine Technology \& Economy, No.70 Xueyuan South Road, Haidian District, Beijing, China \\ ${ }^{*}$ Corresponding author
}

\begin{abstract}
As the continuous development of wireless sensor networks, the network has put forward higher requirements for the transmission of large amounts of data. In order to meet the high efficiency of network transmission, we often use various technologies to increase the transmission rate, but this may also cause high packet loss rate on the network. Therefore, in order to reduce the packet loss rate of the network, this paper proposes a lost packet retransmission technology based on reliable transmission of large amounts of data in wireless sensor networks. This technology is based on a multi-hop efficient transmission network platform composed of multiple wireless nodes. This is an FPGA-based lost packet retransmission mechanism that can make full use of the parallel processing capabilities of the FPGA to reduce the packet loss rate when transmitting large amounts of data. Finally, the experimental results show that this technology can guarantee the reliable transmission of large amounts of data in multi-hop transmission networks and has a lower packet loss rate.
\end{abstract}

Keywords-wireless sensor network (WSN); large amount of data transmission; packet loss rate; lost packet retransmission mechanism

\section{INTRODUCTION}

Wireless sensor networks have been paid more attention and research in recent years due to their advantages of simple implementation and low cost [1]. They are currently widely used in distributed monitoring and testing. When a wireless sensor network monitors a certain area or performs parameter testing on a certain moving object, it can deploy sensor nodes throughout the monitoring or testing area, and the nodes can self-organize and have the ability to transmit data to the outside world [2, 3].

Whether it is a distributed environment security monitoring network or a triggered parametric test network, it needs to perform large data transmission tasks during monitoring or testing. In order to ensure the stability and effectiveness of the network work, it is crucial that large amounts of data are transmitted in complete and efficient manner. Due to the large amount of data, the transmission rate of traditional networks cannot meet the requirements of high data throughput, and it is difficult to achieve sudden large data transmission tasks. Therefore, people have continued to study how to increase the transmission rate of large data transmissions to meet the network requirements in recent years. However, it is considered that the reliability requirement of the test network is high, and the application environment is rather harsh and complex. The wireless link is easily subject to external interference, and packet loss is likely to occur $[4,5]$. Loss of large amounts of data will not only affect the integrity of the test data, but will also reduce the value of its analysis, and thus affect the test results [6].

The commonly used retransmission method is fixed retransmission, and most protocols and standard definitions need to confirm each packet of data [7]. However, this retransmission mechanism has many data packet transmission times and long retransmission time, which greatly reduces the transmission efficiency of the large data transmission network. In view of the above problems, this paper proposes a new lost packet retransmission mechanism based on burst large data volume multi-hop high-speed transmission network. The mechanism is based on a point-to-point multi-hop high-speed wireless transmission network system. The network has a high transmission rate, but it has a certain packet loss rate. The lost packet transmission mechanism is based on the parallel processing capability of the FPGA controller to achieve packet number statistics for lost packets, the packets are sent to the sending node after the large data volume is transmitted and the sending node uses the packet number to perform packet query. And send the data to the receiving node.

\section{HARDWARE DESIGN}

The wireless transmission network is a multi-hop selforganizing network composed of a plurality of wireless nodes. The block diagram of the wireless node is shown in Fig. 1. It is mainly composed of the FPGA master control chip, external data interface, wireless module, clock module, power management module, SDRAM memory, Flash memory, and micro-SD card etc. Among them, the power management module provides power to the entire node, the clock module provides the control clock, FPGA is the main controller of the whole node, the external data interface finishes the data acquisition, SDRAM memory, Flash memory and micro-SD card realize the preservation of the procedure and data and the wireless module is responsible for the wireless transmission of data. 


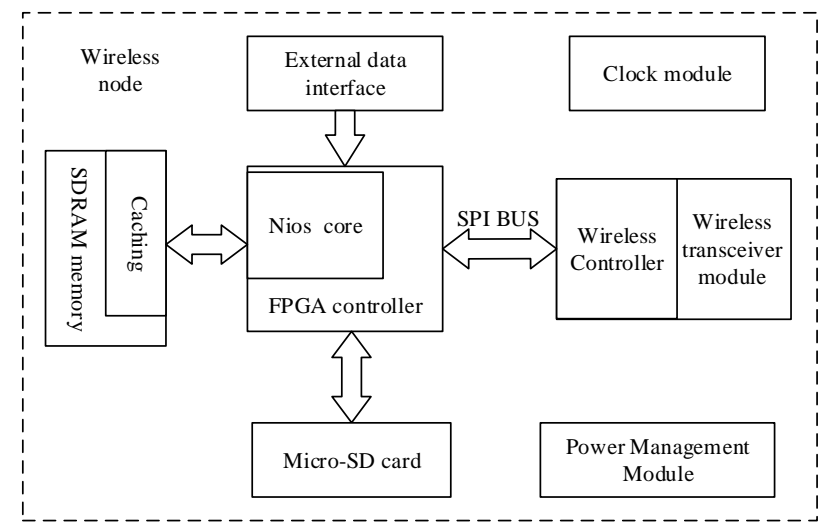

FIGURE I. THE BLOCK DIAGRAM OF WIRELESS NODE

The node collects a large amount of data through the external data interface and transmits it to the main control chip FPGA, and then realizes the processing and storage of big data inside the FPGA. Data processing inside the node is mainly realized by hardware IP cores, packet identification, packet number parsing and lost packet statistics. Data storage is mainly implemented by hardware, and the NIOS core controls storage parameters. The hardware IP core performs streamlined processing of high-speed data streams, verification, response, interface protocol adaptation, backup, and other processing operations. The NIOS core mainly implements control operations such as status monitoring of data stream processing and parameter adjustment.

In terms of wireless data transmission, a wireless module integrated with a wireless controller and a wireless transceiver module is used. The communication between the FPGA and the wireless module is via the SPI bus, and the commands and data exchange between the two are implemented. The wireless transmission protocol is programmed on the wireless controller to control the sending and receiving of the wireless transceiver module.

\section{LOST PACKET RETRANSMISSION MECHANISM}

\section{A. Problem Analysis}

Compared with wired transmission networks, wireless transmission networks always have a certain packet loss rate. With the continuous development of wireless sensor networks, the demand for networks with low packet loss rate is increasing in most areas. In addition, external factors such as harsh environment or long-distance transmission have large interference to the network transmission link, which causes a sharp increase in the packet loss rate.

Lost packet retransmission is one of the effective means to reduce the packet loss rate of the network. The commonly used lost packet retransmission mode is fixed retransmission. As shown in Fig. 2, each data packet transmission defined in the method needs to be confirmed. That is, one sending process consists of two parts: sending the data packet and receiving the ACK signal. Whether the data packet is transmitted successfully or not depends on whether an ACK is received or not. If the data packet sending node does not receive the ACK signal from the data packet receiving node, the sending node will consider the data packet lost after timeout, and then retransmit the data packet until an ACK signal is received.

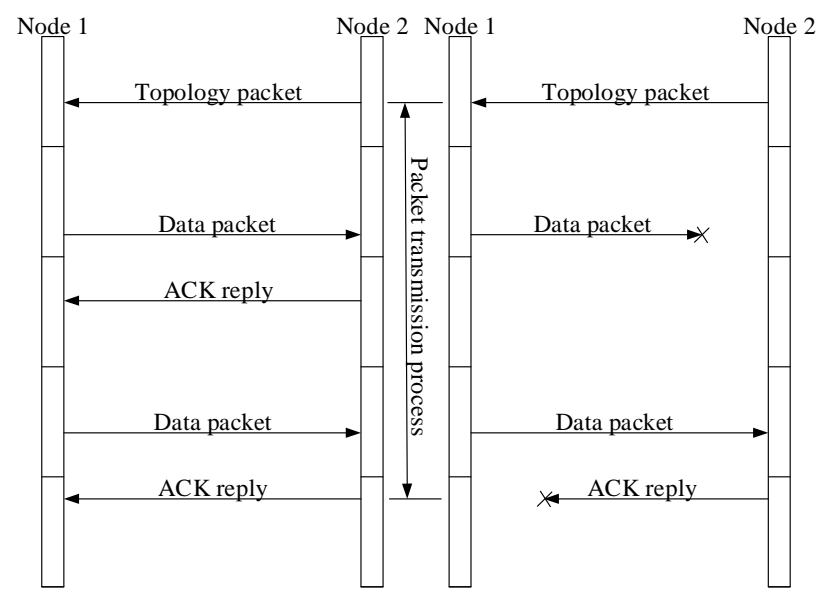

(a)No retransmission situation

(b)Retransmission situation

FIGURE II. TRADITIONAL LOST PACKET RETRANSMISSION

In the conventional lost packet retransmission mode, each data packet needs to determine whether to retransmit the data packet or to send the next data packet according to whether the reply ACK signal is received or not. First of all, the data packet loss rate is high due to the large amount of data and high transmission rate in a large data high-speed transmission network. If this lost packet retransmission method is adopted, the efficiency of data transmission will be greatly reduced. Secondly, because of the irregularity and vulnerability of wireless network transmission links, there may be two reasons why a sending node cannot receive an ACK signal. The first possibility is that the data packet itself is lost during transmission, and the receiving node does not receive the data packet at all, an ACK signal cannot be sent, and the sending node naturally cannot receive the ACK reply. The second possibility is that the receiving node receives the data packet and sends out an ACK signal, but the ACK signal is lost during the transmission, and at this time Nodes also cannot receive ACK replies. Therefore, not only the time slots for retransmission of a single data are long, but also the probability of retransmission of the data packet is increased in the data transmission process. This will cause the entire network to be unreliable and unable to adapt to the high-speed transmission of a large amount of data. Therefore, this paper proposes a new type of lost packet retransmission mechanism based on hardware acceleration.

\section{B. Lost Packet Retransmission Mechanism}

In order to change the inefficiency of traditional lost packet retransmission methods, this paper designs a new type of lost packet retransmission mechanism based on hardware acceleration. According to the low efficiency of traditional retransmission methods, this new lost packet retransmission mechanism is designed not to use each packet based on whether it receives an ACK signal to determine whether it needs retransmission. Instead, adopting data and packet loss. Transmission method separately. The overall transmission process is shown in Fig. 3. In this lost packet retransmission 
mechanism, data is continuously transmitted without packet loss judgment and retransmission during packet transmission. Lost packet detection and statistics are performed. After the data transmission ends, if the receiving node detects and statistics the lost data packet, it will send the lost packet number to the sending node. Then the sending node finds the data of the corresponding packet number in the local storage and sends them to the receiving node. Of course, packet loss is likely to occur in the process of lost packet retransmission. However, these lost packet are minimal, compared to the amount of lost packets due to the large amount of data transmission. As a result, this reduces the system packet loss rate, but does not reduce the network's high efficiency.

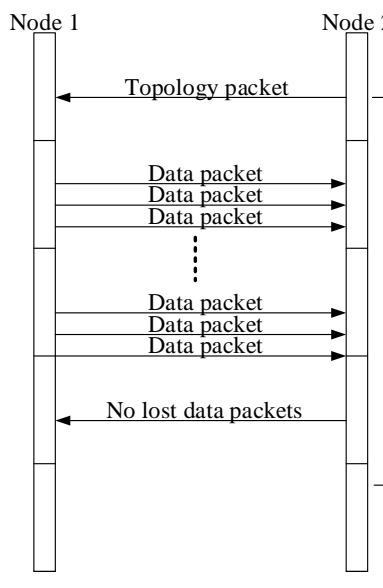

(a)No retransmission situation

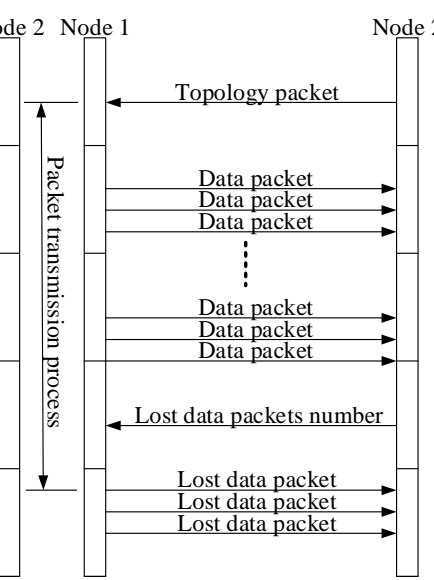

(b)Retransmission situation
FIGURE III. NEW LOST PACKET RETRANSMISSION

The flow chart of the entire lost packet retransmission mechanism is shown in Fig. 4. The lost packet retransmission mechanism is mainly divided into three parts: lost packet number statistics, the lost packet query and the lost packet retransmission. The packet loss number statistics are transmitted when the large amount of data is transmitted. Since the transmission speed of large amount of data transmission is high, the packet number statistics must be performed quickly to avoid reducing the data transmission rate. In general, hardware implementations are faster than software implementations. In hardware design, FPGA is favored for its ability of parallel processing, so the packet loss number statistics is based on FPGA hardware. After the large data volume transmission is completed, the packet number statistics are also completed. At this time, the receiving node sends the packet numbers that are counted to the sending node. After receiving the packet number, the receiving node queries the lost packet. The process is implemented in the NIOS core through the $\mathrm{C}$ language, according to the contents of the packet number to query the contents of the packet corresponding to the packet number in the micro-SD card. After querying all the lost data packets, the sending node sends a lost packet retransmission command to the receiving node. The receiving node enters a cyclic receiving mode after receiving the command. The lost packet transmission is the same as the previous large data volume transmission at this time. The retransmission mechanism may reduce the transmission rate of the entire network, but it also greatly reduces the packet loss rate of the network. Therefore, the transmission rate and packet loss rate are weighed, and the retransmission mechanism is applicable in a wireless sensor network with a large amount of data transmission.

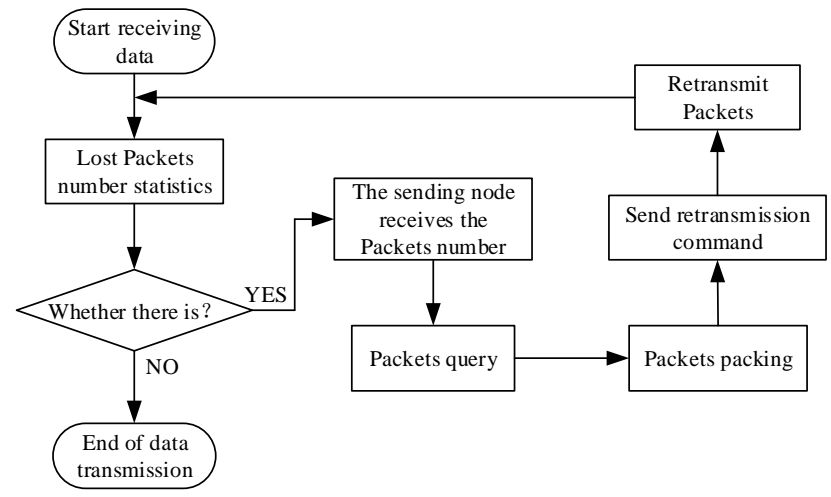

FIGURE IV. THE FLOW CHART OF THE ENTIRE LOST PACKET RETRANSMISSION MECHANISM

Packets all have packet headers of sequential packet numbers in the transmission network, so the packet numbers are continuous in the process of receiving data packets. As shown in Fig. 5, the main idea of the lost packet number statistics is to judge how many data packets are lost by judging the difference between two adjacent packet numbers.

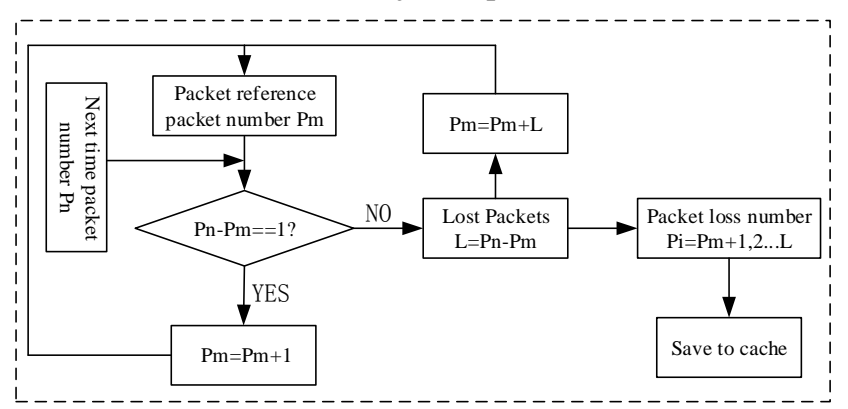

FIGURE V. LOST PACKET STATISTICS ALGORITHM

Assuming that the packet number of the first detection is $P_{m}$ and the packet number of the second detection is $P_{n}$, the number of lost packets is:

$$
L=P_{n}-P_{m}
$$

And the packet number of lost $L$ packets are:

$$
P=P_{\mathrm{m}}+i \quad(i=1,2 \ldots L)
$$

After the data transmission is completed, the data receiving node saves the lost packet numbers that are counted in the cache and sends them to the sending node. After the sending node receives the number of the lost packet, it performs data packet query according to the packet number within the FPGA. The main idea of the packet query is to use the packet number 
and the first packet number $P_{0}$ stored in the micro-SD card of the node to make a difference. This difference is used as the offset when querying to perform packet query. The first data packet number plus the offset is used to accurately find the data packet when reading the data packet in the micro-SD card:

$$
\Delta=P-P_{0}
$$

Finally, the data sending node sends the lost data packets that is queried to the receiving node. After receiving the data packets, the receiving node will sort and store the data according to the data packet number to facilitate the next hop data transmission.

\section{EXPERIMENTAL RESULTS}

In order to verify the correctness of the lost packet retransmission mechanism, this paper designs an experiment for the retransmission mechanism. The function of the retransmission mechanism is tested by high-speed transmission of large amounts of data between point-to-point nodes.

The host computer continuously sends the data packets to the data sending node through the serial port, and the data packets are accumulated in sequence by the data sending node. After the internal data packets are processed and stored, the data packets are sent to the data receiving node, and are transmitted during the entire data packet transmission process. The information of packet loss detection and packet loss retransmission is displayed through the serial port to the serial debugging assistant. The test display information is shown in Fig. 6. The reliability of the lost packet retransmission mechanism is determined by the displayed information and the data stored in the micro-SD card.

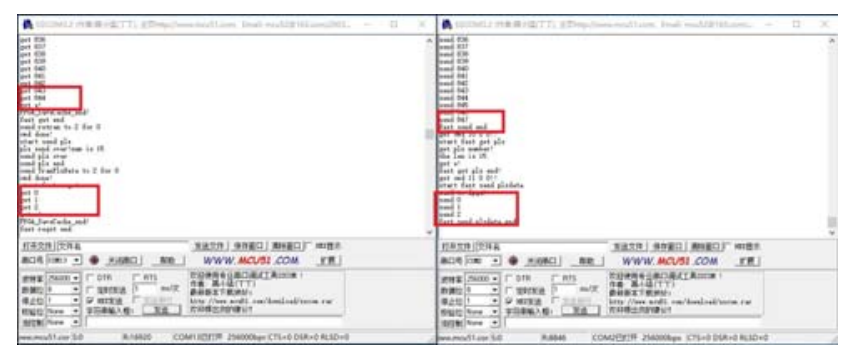

FIGURE VI. THE TEST DISPLAY INFORMATION

As can be seen from the figure, node 2 sent 847 packets to node 1 and node 1 received only 844 packets, indicating that there were 3 lost packets. Then through the packet loss and retransmission mechanism, node 1 counts the packet numbers of the three packets and sends the packet numbers to node 2 . Then, node 2 looks up these packets according to the packet numbers and sends them to Node 1 , and Node 1 also received these 3 packets, achieving 100\% loss of packet retransmissions. It can be seen that the mechanism is able to achieve the predetermined function.

\section{SUMMARY}

This paper proposes a lost packet retransmission technology based on large data transmission in wireless sensor networks, which achieves a low packet loss rate for large data transmission networks. The experimental results show that lost packet retransmission mechanism based on hardware acceleration can effectively reduce the packet loss rate during the transmission of large data volume. The retransmission mechanism not only overcomes the inefficiency of the traditional dropout retransmission mechanism, but also avoids the risk of high packet loss rates, which make it more suitable for most transporting networks.

However, the lost packet retransmission mechanism loses the transmission rate to a certain extent. With the increase of the hop count, the transmission rate loss becomes more obvious. Therefore, more data compression and compression sensing may be used in the future research on data integrity of largescale data transmission in wireless sensor networks. This can not only effectively increase the transmission rate of the network, but also can structure the complete data, which makes the entire wireless sensor network more efficient and reliable.

\section{ACKNOWLEDGMENT}

This work was partially supported by the National Basic Scientific Research Program of China (JCKY2016208B007). We are also very grateful to the reviewers for their useful opinions and suggestions, which have improved representativeness.

\section{REFERENCES}

[1] Fang-Min L I, Ping H, Luo T. Adaptive area location algorithm combining with packet lost rate and RSSI in wireless sensor networks[J]. Journal on Communications, 2009, 30(9):15-23.

[2] AKYILDIZ I F, KASIMOGLU I H. Wireless sensor and actor networks: research challenges [J]. Ad Hoc Networks Journal, 2004, 2(4): 351-367.J. Clerk Maxwell, A Treatise on Electricity and Magnetism, 3rd ed., vol. 2. Oxford: Clarendon, 1892, pp.68-73.

[3] SUZUKI M, SARUWATARI S, KURATA N, et al. A high-density earthquake monitoring system using wireless sensor networks[C]// A high-density earthquake monitoring system using wireless sensor networks. International Conference on Embedded Networked Sensor Systems, SENSYS 2007, Sydney, Nsw, Australia, November. 373-374.

[4] Guo Z Q, Wang Q, Wan Y D, et al. Modeling of burstiness for IEEE 802.15.4 radio link and optimizing for retransmission mechanism in industrial environments[J]. Ruan Jian Xue Bao/journal of Software, 2012, 23(SUPPL.).

[5] Gungor V C, Hancke G P. Industrial Wireless Sensor Networks: Challenges, Design Principles, and Technical Approaches[J]. IEEE Transactions on Industrial Electronics, 2009, 56(10):4258-4265.

[6] Chen G S. Research on Data Loss and Recovery of Sensor Networks Based on Compressed Sensing[D]. Shanghai Jiao Tong University, 2014.

[7] Wan Y, Wang Q, Xiaotong A Z. An Invited Retransmitting Method for Cluster based Wireless Sensor Network[J]. Journal of Computer Research \& Development, 2011. 